\title{
An exploration of the genetic and environmental etiology of heart rate in infancy and middle childhood
}

\author{
Carol A Van Hulle ${ }^{1,4}$, Robin Corley ${ }^{1}$, Carolyn Zahn-Waxler ${ }^{2}$, Jerome Kagan ${ }^{3}$ and John K Hewitt ${ }^{1}$ \\ ${ }^{1}$ Institute for Behavioral Genetics, University of Colorado, Boulder, CO \\ ${ }^{2}$ National Institutes of Mental Health, Bethesda, MD \\ ${ }^{3}$ Department of Psychology, Harvard University, Cambridge, MA, USA
}

\begin{abstract}
Heart rate was recorded on $210 \mathrm{MZ}$ and $174 \mathrm{DZ}$ same sex twin pairs participating in the MacArthur Longitudinal Twin Study (MALTS) at age 14, 20, 24, 36 months and 7 years. Heart rate was monitored in the laboratory at all ages. At ages 14 to 36 months, heart rate was monitored prior to a set of cognitive tasks. At age 7 years heart rate was recorded during a mood-eliciting videotaped presentation. At this age only heart rate monitored during neutral portions of the presentation were used. Mean heart rate declines substantially across this age range, but is si milar in boys and girls and for MZ and DZ twins at each age. Heart rate is moderately correlated across all time points suggesting that individual differences in heart rate are rel atively stable over this age range. Multivariate genetic and environmental models were fitted to the raw data. In general, genetic factors contribute to the stability of individual differences over time. Shared and nonshared environment factors tended to be occasion specific, with non-shared environment contributing substantially to the individual variation at each age. Shared environment and nonshared environment also contributed a modicum to the stability across time. Thus, individual differences in resting heart rate is a relatively stable, heritable trait from infancy to early childhood. Twin Research (2000) 3, 259-265.
\end{abstract}

Keywords: twins, heart rate, Iongitudinal, childhood, genetics

\section{Introduction}

There is growing interest in heart rate patterns in childhood as predictors of later behavior. ${ }^{1,2}$ Inhibited children for instance were found to have higher, more stable heart rates than uninhibited children, ${ }^{3}$ and children with low heart rates have been reported to have a greater tendency to engage in delinquent behaviour. ${ }^{4}$ The conceptual ization of heart rate as an indicator of temperament would be perhaps more plausible if individual differences in heart rate were shown to be a stable, heritable characteristic. Studies support the stability of individual differences in heart rate for both children and adults. ${ }^{5,6}$ Mathers, Woodall, and Stoney ${ }^{7}$ reported an average correlation of 0.46 for heart rate measured over a 4 year interval on children aged 6-18. Correlations for males were slightly higher than those for females. Fracasso, Porges, Lamb, and Rosenberg ${ }^{8}$ recorded infant heart rate at 5, 7, 10, and 13 months. Correlations over these ages range from 0.32 to 0.57 .

Numerous family and twin studies have provided evidence for a moderate genetic influence in heart

Correspondence: Carole A Van Hulle, Institute for Behavioral Genetics, CB 447, University of Colorado, Boulder, CO 80309-0447, USA. Tel: (303)4921475; Fax: (303) 482 8063; E-mail: vanhulle@colorado.edu

Received 7 February 2000; revised 22 March 2000; accepted 7 April 2000 rate. ${ }^{9-13}$ Singh, ${ }^{14}$ for instance, compared sibling and spousal correlations in the Framingham Heart Study and suggested that genetic factors accounted for $21 \%$ of the variance. Most twin studies that focus on adolescent and adult populations report higher heritabilities of approximately $0.5 .^{15-17}$ One study of 11-year-old twins also found that genetic factors contributed to $50 \%$ of the variance. ${ }^{18}$ However, another study which included younger twins, aged 5-12 years, reported a lower heritability of $0.37 .^{19}$ Modeling techniques allow one to move beyond simply estimating heritabilities from twin correlations to determining the role of genetic, shared environmental and non-shared environmental influences on a phenotypic trait and its development. Boomsma ${ }^{20}$ fitted genetic model s to resting heart rate obtained from adolescents (aged 14-20 years). Again, heritable factors were found to account for approximately $50 \%$ of the individual variation in resting heart rate. Shared and non-shared environmental factors contributed about a quarter each to the remaining variation. However, several other studies on both adults and children failed to find any effect for shared environment. ${ }^{16-18}$ Rather, genetic and non-shared environment each contribute about equally to the individual variation in resting heart rate.

Whilst there have been studies on the stability of heart rate in young children there are few studies on 
the heritability of heart rate at early ages. Also, there are few studies that examine the heritability of heart rate over time. The purpose of this study is to determine if individual differences are stable from infancy to middle childhood and, if so, to what extent the stability is due to genetic or environmental factors, and how these factors change across time. Women tend to have faster resting heart rates than men. ${ }^{21} \mathrm{Also}$, it is well known that heart rate is inversely related to body size developmentally and across species, ${ }^{22}$ and both height and weight are highly heritable. ${ }^{23}$ Therefore the effect of these possible confounding variables on the etiology of heart rate are also considered.

\section{Method \\ Subjects}

The sample consisted of 210 monozygotic (MZ) and 174 dizygotic (DZ) same sex twin pairs from the MacArthur Longitudinal Twin Study (MALTS). There were 105 male and female $M Z$ pairs, 93 male DZ pairs and 81 female DZ pairs. MALTS was initiated in 1986 and enrollment lasted until the end of 1990. During this time the Colorado Department of Heal th contacted all twins born in the state; $50 \%$ of eligible families agreed to take part in twin studies. Families were selected preferentially for twin births that met the criterion of having a birth weight of $1700 \mathrm{~g}$ or more, a gestational age of at least 34 weeks, and excluding those with medical complications. Over $90 \%$ of the sample is Caucasian, of which $8 \%$ is Hispanic. Biases in demographic variables between the subject sample and the general population were small and there was no selective attrition over time. Zygosity was initially determined using a questionnaire based on the diagnostic rules developed by Nichols and Bilbro ${ }^{24}$ and, in most cases, zygosities have been confirmed by genotyping. The actual sample size at each age ranges from 442 to 573 subjects; missing data occurred because of equipment failure, excessive noise in the ECG signal, and subject non-participation on one or more occasions.

\section{Procedures}

Heart rates were recorded in the laboratory at age 14, 20,24 and 36 months and at age 7 years. At ages 14 , 20 , and 24 months subjects' heart rates were monitored for $90 \mathrm{~s}$ prior to a set of cognitive tasks during which six slides were presented for $15 \mathrm{~s}$ each (pretask). Heart rate was monitored again for two minutes on completion of word comprehension, categorization, and memory locations tasks (posttask). At age 36 months heart rate was monitored for two minutes prior to and upon completion of a set of cognitive tasks. Because of the high correlations between pre-task and post-task heart rate ( $r$ ranged from 0.75 to 0.82 ) at these ages the heart rate used in the present analysis is the average across pre-task and post-task conditions. At age 7 years, heart rate was recorded while subjects viewed the Mood Induction Stimulus for Children, ${ }^{25}$ a videotaped presentation designed to present mood inducing stimuli. Each $60 \mathrm{~s}$ mood-inducing episode was preceded by a 15 s neutral event. ECG signal was used to determine inter-beat intervals (IBI), which represent milliseconds between successive R-waves. The R-wave onset times were scored automatically and checked manually. A mean IBI was calculated for each of the nine neutral events. Heart rate for age 7 years was calculated as the mean IBI across all available neutral events, and converted to beats per minute prior to further analysis.

\section{Model fitting}

The purpose of this study is to explore the contributions of genes and environment to the individual differences in heart rate and to the change and continuity of heart rate from infancy to middle childhood. To that end, a sequence of models was fitted to the raw data, beginning with a fully parameterized model and proceeding to more parsimonious models proposed in the developmental genetic literature. For each class of models three sources of variation, genetic, shared environmental and non-shared environmental, were considered and a series of submodels were tested to arrive at the most parsimonious explanation of the data. In order to make comparisons between competing models it was necessary to establish a baseline model for raw data. To do so, a model which made no assumptions about the genetic and environmental contributions to the variance was fitted to the 10 heart rate observations for each twin pair. For each zygosity, this fully saturated model allowed for 55 parameters to specify the expected variances and covariances among the observations. Instead of estimating the means for each zygosity as well, in this and all subsequent models, the mean values for each zygosity group were set to the observed means for that group. All models were fitted to the unstandardized observations by Maximum Likelihood Estimation implemented in the program $M x .{ }^{26}$ When fitting to raw data, the log likelihood function maximized is

$$
\mathrm{LL}=\sum_{\mathrm{i}=1}^{\mathrm{n}}\left[-\frac{1}{2}\left|\Sigma_{\mathrm{i}}\right|-\frac{1}{2}\left(\mathbf{x}_{\mathrm{i}}-\mu\right)^{\mathrm{t}} \Sigma^{-1}\left(\mathbf{x}_{\mathrm{i}}-\mu\right)\right]
$$

In this equation, $\Sigma_{\mathrm{i}}$ refers to the expected covariance matrix appropriate for the twin type, $x_{i}$ is a vector of 
observations, and $\mu$ is a vector of means for the twin type.

Next a Chol esky decomposition was fit to the data. A Cholesky decomposition is a series of factor models that saturate the observed variances and covariances. That is, for each source of variation, genetic $\left(a^{2}\right)$, shared environment $\left(c^{2}\right)$, and nonshared environment $\left(e^{2}\right), 15$ free parameters are necessary to specify the variances and covariances in heart rate measured over the five ages. This yields a total of 45 parameters. The expected covariance matrix is defined as

$$
\Sigma=\left\{\begin{array}{ll}
G+C+E & r \otimes G+C \\
r \otimes G+C & G+C+E
\end{array}\right\}
$$

where $G$, the additive genetic variance, is a product of the matrix of path coefficients from the latent genetic factor to the phenotype and its transpose, C is the shared environmental variance, $E$ is the nonshared environmental variance, and $r$ is the additive genetic correlation (1.0 for MZ twins and 0.5 for DZ twins). A significantly worse fit relative to the baseline model would indicate a failure of the assumption that the variances and covariances among the measurements are the same for all individuals in the study, irrespective of zygosity or designation as first or second twin, and/or a failure of the assumption that DZ twin pair covariances are not greater than $M Z$ twin pair covariances.

Common Pathway, Independent Pathways, and Simplex models were also fitted to the data. Each model makes different assumptions about how latent factors influence the development of the phenotype. In a Common Pathway model the covariation between ages results from a single latent phenotypic variable determined by genetic and environmental factors; this latent phenotype has a direct effect on each of the five heart rate measurements. The variation at each age is determined by the common latent phenotype together with age-specific genetic and environmental factors. Thus, only 23 parameters are required to account for the variances and co-variances of the five heart rate measurements. In the Independent Pathways model, covariation between ages is determined by separate genetic, shared environmental, and non-shared environmental factors common to heart rate at all five ages. Variance in heart rate at each age is again determined by the factors common across ages together with genetic and environmental factors specific to each age resulting in 20 specified parameters. In the Simplex model covariances among the five ages of measurement are specified by genetic and environmental factors specific to each age and the 'carryover effects' or transmission of these factors to subsequent ages. The variance is a product of the age-specific effects and age-to-age transmission. Hence, the additive genetic covariance matrix is

$$
\mathrm{G}=\left(I-\beta_{\mathrm{G}}\right)^{-1} \Psi_{\mathrm{G}}\left(I-\beta_{\mathrm{G}}^{\prime}\right)^{-1}
$$

where genetic transmission parameters are modeled in matrix $\beta_{\mathrm{G}}$, a $5 \times 5$ matrix with four transmission parameters on its subdiagonal, and $\Psi_{G}$ is a $5 \times 5$ diagonal matrix of age-specific variances. Similar parameter matrices can be defined for the other sources of variation giving a total of 27 parameters.

\section{Results}

The means and standard deviations for $M Z$ and $D Z$ twins, pooled across genders, are presented in Table 1. Girls had faster average heart rates than boys al though the mean differences did not exceed 2 beats per minute (bpm) and only reached significance at age 24 months. Mean differences between $M Z$ and DZ twins also did not exceed $2 \mathrm{bpm}$, and there was no effect for twin order. Three outliers more than four standard deviations from the group mean for the same age and gender were excluded from the analysis. Heart rate decreased markedly from approximately $134 \mathrm{bpm}$ at age 14 months to $83 \mathrm{bpm}$ at age 7 years. A repeated measures ANOVA indicated significant decreases in heart rate across ages. However, the individual differences in heart rate remain modestly stable, as can be seen from the correlations among the five ages of measurements (see Table2). Correlations for each age calculated separately for $M Z$ and $D Z$ twins are shown in

Table 1 Means and standard deviations for heart rate

\begin{tabular}{llrrlrr}
\hline & \multicolumn{3}{c}{ MZ twins } & \multicolumn{3}{c}{ DZ twins } \\
Age & N & Mean & S.D. & N & Mean & S.D. \\
\hline 14 months & 318 & 134.03 & 9.87 & 255 & 133.48 & 10.12 \\
20 months & 281 & 129.78 & 9.34 & 244 & 128.27 & 9.55 \\
24 months & 289 & 126.30 & 9.09 & 260 & 126.09 & 9.71 \\
36 months & 243 & 117.21 & 10.11 & 199 & 118.15 & 10.73 \\
7 years & 233 & 81.72 & 9.29 & 204 & 83.82 & 9.66 \\
\hline
\end{tabular}

Table 2 Correlations between heart rate at each age

\begin{tabular}{lllll}
\hline & 14 months & 20 months & 24 months & 36 months \\
\hline 20 months & 0.39 & & & \\
& $(429)$ & & & \\
24 months & 0.33 & 0.44 & & \\
& $(438)$ & $(445)$ & & \\
36 months & 0.33 & 0.34 & 0.49 & \\
& $(344)$ & $(344)$ & $(358)$ & 0.48 \\
7 years & 0.30 & 0.26 & 0.29 & $(267)$ \\
& $(325)$ & $(311)$ & $(329)$ & \\
\hline
\end{tabular}

Sample sizes are in parentheses. All correlations are significant at the 0.001 level. 
Table $3 \mathrm{MZ}$ and DZ twin correlations for heart rate at each age

\begin{tabular}{lll}
\hline & MZ twins & DZ twins \\
\hline 14 months & 0.58 & 0.49 \\
& $(139)$ & $(112)$ \\
20 months & 0.46 & 0.40 \\
& $(130)$ & $(113)$ \\
24 months & 0.64 & 0.36 \\
& $(135)$ & $(123)$ \\
36 months & 0.49 & 0.42 \\
7 years & $(106)$ & $(84)$ \\
& 0.65 & 0.44 \\
& $(111)$ & $(98)$ \\
\hline
\end{tabular}

Sample sizes are in parentheses. All correlations are significant at the 0.001 level.

Table 3. At each age of measurement the MZ correlation is higher than the DZ correlation suggesting genetic influences on heart rate. However, the high DZ correlations relative to half the MZ correlations suggest that shared environment influences heart rate as well. Finally, the moderate $M Z$ correlations, less than unity, indicate the influence of non-shared environment and unreliability.

The results of the model fitting are presented in Table4. The adequacy of any model compared with the fully saturated baseline model can be determined by a likelihood ratio $\chi^{2}$ test derived from the differences in log-likelihoods. As noted above there are small but occasionally significant gender differences. An omnibus test of the heterogeneity of means and covariances between the genders, in which a baseline model that constrained means and covariances to be the same across genders was compared with a model that allowed for different means and covariances for each gender, proved to be nonsignificant $\left(\Delta \chi_{130}^{2}=154.14, P=0.07\right)$. Therefore, the analyses presented below were performed on pooled data. As can be seen from Table4, the Cholesky decomposition did not produce a significantly worse fit than the baseline model $(P=0.09)$, though it is much more parsimonious. An examination of the path coefficients suggests the presence of a single genetic factor common to all ages and, to a lesser extent, a single shared environmental factor common across ages. Non-shared environmental factors seem specific to each age.

Of the more theoretical models, only the Common Pathway model failed to fit the data $(P=0.003)$. Both the Independent Pathways and Simplex models did not provide a significantly worse fit compared with the baseline model ( $P=0.12$ and 0.11 , respectively). Inspection of the parameter estimates from the Independent Pathways model suggested that genetic factors common across ages contributed more to individual variation than genetic factors specific to each age. Conversely, environmental factors specific to each age appeared to contribute more to the individual variation than environmental factors common to all ages. Several submodels were fitted to the data. The fit of these submodels relative to the full Independent Pathways model are presented in Table 5. As suspected, dropping the common genetic factor from the model caused the model to fail $(P<0.001)$. The Akaike's Information Criterion (AIC), $\chi^{2}-2 \mathrm{df}$, is often used as a measure of the most parsimonious description of the data. Inspection of the AIC values reveal ed the best fitting submodel to be that in which only those genetic factors specific to each age were dropped $(A I C=-70.35)$. Similar results were obtained from the Simplex model. As before, several sub-models were fitted to the data. Their fit relative to the full Simplex model is also presented in Table5. Once again a model in which genetic transmission factors were dropped fitted significantly worse than the full model $(P<0.001)$. Also, the sub-model with the lowest AIC value again excluded genetic effects specific to each age (AIC = 71.14). The reduced Independent Pathways and Simplex models are presented with their standardized path coefficients in Figures 1 and 2 respectively. The estimated proportions of genetic, shared environmental and non-shared environmental variances based on the most parsimonious Independent Pathways and Simplex submodels are presented in Table6.

The possible confounding effect of body size was also explored. Since both height and weight are heritable and are related to heart rate, it was desirable to determine if the genetic influences in heart rate existed above and beyond individual differences in height and weight. Boys were significantly heavier than girls at all ages except age 7 years and were significantly taller except at age 22 months and at age 7 years. Heart rate at each time point was regressed on gender, height, weight and the interactions of height and weight with gender. In general height and weight only accounted for 3-5\% of the variance in heart rate. The residuals were fitted to a Cholesky decomposition and compared

Table 4 Model comparisons

\begin{tabular}{|c|c|c|c|c|c|}
\hline Model & $-2 * \mathrm{LL}$ & $\Delta x^{2}$ & $\triangle \mathrm{df}$ & P-value & $\mathrm{AIC}$ \\
\hline Baseline model & 17953.61 & & & & \\
\hline Full Cholesky decomposition & 18034.37 & 80.76 & 65 & 0.09 & -49.24 \\
\hline Full common pathway model & 18083.13 & 129.53 & 87 & 0.003 & -46.47 \\
\hline Full independent pathways model & 18048.74 & 95.13 & 80 & 0.12 & -64.87 \\
\hline Full simplex model & 18053.04 & 99.43 & 83 & 0.11 & -66.57 \\
\hline
\end{tabular}


with the fully saturated baseline model. The Cholesky decomposition produced a significantly worse fit than the baseline model $(P=0.03)$. However, the standardized estimates derived from fitting a Cholesky decomposition to raw heart rate data and those derived from fitting a Cholesky decomposition to the residual heart rate data show a high degree of agreement. Borrowing from factor analysis, a coefficient of congruence was cal culated between the two sets of estimates according to the formula

$$
\frac{\sum l_{i 1} \cdot l_{i 2}}{\sum l_{i 1}^{2} \cdot l_{i 2}^{2}}
$$

where $I_{i 1}$ is the ith path coefficient from the first solution and $\mathrm{I}_{\mathrm{i} 2}$ is the corresponding coefficient from the second solution. The coefficient of congruence was 0.98 for the genetic and non-shared environment loadings, and 0.90 for the shared environment loadings. Therefore, the pattern of influences on heart rate that we have described appear to be largely independent of body size.

\section{Discussion}

Several models were found to fit the data adequately; the best-fitting models excluded genetic effects specific to each age. Longitudinal data often conform to a Simplex structure, that is, correlations are highest between adjacent time points and decrease systematically as the distance between time points increases, and are best explained by the Simplex model as described above. However, when the transmission parameters of the Simplex model approach unity, as they do here for the genetic effects, then the expectations based on the model become indistinguishable from those based on an Independent Pathways model. This suggests that either the Simplex or the Independent Pathways submodels that exclude age specific genetic effects give essentially equival ent explanations of the data. From Figures 1 and 2 and Table 6, it can be seen that both models show that stability across ages in individual differences is largely due to a single genetic factor. However, each model indicates a slightly different pattern of influence for shared and non-shared environments. In the Independent Pathways model shared environment contributes moderately to individual variation at each age but, except for small effects at ages 14 and 20 months, does not contribute much to the stability across time. The opposite pattern is evident in the Simplex model. Again shared environment contributes moderately to the individual variation within a given age. However, there are moderate shared-environmental transmission effects at ages 36 months and 7 years. Nonshared environment contributes as much as $28 \%$ of the covariance in the Independent Pathways model, while contributing al most nothing to the stability of individual variation over time in the Simplex model.

In general, non-shared environment contributed $30 \%$ to $50 \%$ of the individual variation. Of the remaining variance, genetic factors accounted for slightly more than shared environment. Genetic factors contributed about $20-50 \%$ toward the total variance and shared environment influences contributed $10-40 \%$ to the total variance. In this sample the genetic factors influencing heart rate do not appear to be mediated by body size, although few children fell into the extreme ranges for body size.

Interest in resting heart rate stems from reports that heart rate is an important indicator of certain

Table 5 Comparison among submodels

\begin{tabular}{|c|c|c|c|c|c|}
\hline Model & $-2 * \mathrm{LL}$ & $\Delta x^{2}$ & $\triangle \mathrm{df}$ & P-value & $\mathrm{AIC}$ \\
\hline $\begin{array}{l}\text { Full independent pathways model } \\
\text { Dropped age specific } a^{2} \\
\text { Dropped age specific } c^{2} \\
\text { Dropped common } a^{2} \\
\text { Dropped common } c^{2} \\
\text { Dropped common } e^{2} \\
\text { Dropped age specific } a^{2} \text { and common } e^{2} \\
\text { Dropped age specific } a^{2} \text { and common } c^{2} \\
\text { Dropped age specific } a^{2} \text { and specific } c^{2}\end{array}$ & 18048.74 & $\begin{array}{r}4.52 \\
10.08 \\
30.51 \\
11.96 \\
17.14 \\
19.90 \\
18.74 \\
64.53\end{array}$ & $\begin{array}{r}5 \\
5 \\
5 \\
5 \\
5 \\
10 \\
10 \\
10\end{array}$ & $\begin{array}{c}0.47 \\
0.07 \\
<0.001 \\
0.03 \\
0.004 \\
0.03 \\
0.04 \\
<0.001\end{array}$ & $\begin{array}{r}-70.35 \\
-64.70 \\
20.52 \\
-62.90 \\
-57.70 \\
-64.90 \\
-66.13 \\
-20.34\end{array}$ \\
\hline $\begin{array}{l}\text { Full simplex model } \\
\text { Dropped age specific } \mathrm{a}^{2} \\
\text { Dropped age specific } c^{2} \\
\text { Dropped common } \mathrm{a}^{2} \\
\text { Dropped common } \mathrm{c}^{2} \\
\text { Dropped common } \mathrm{e}^{2} \\
\text { Dropped age specific } \mathrm{a}^{2} \text { and common } \mathrm{e}^{2} \\
\text { Dropped age specific } \mathrm{a}^{2} \text { and common } c^{2} \\
\text { Dropped age specific } \mathrm{c}^{2} \text { and common } \mathrm{e}^{2}\end{array}$ & 18053.04 & $\begin{array}{r}3.46 \\
7.89 \\
111.99 \\
8.76 \\
7.25 \\
11.48 \\
20.9 \\
14.68\end{array}$ & $\begin{array}{l}4 \\
4 \\
4 \\
4 \\
4 \\
8 \\
8 \\
8\end{array}$ & $\begin{array}{l}0.48 \\
0.09 \\
<0.001 \\
0.06 \\
0.12 \\
0.18 \\
0.007 \\
0.06\end{array}$ & $\begin{array}{r}-71.14 \\
-66.67 \\
37.59 \\
-65.81 \\
-67.31 \\
-71.09 \\
-61.66 \\
-67.89\end{array}$ \\
\hline
\end{tabular}




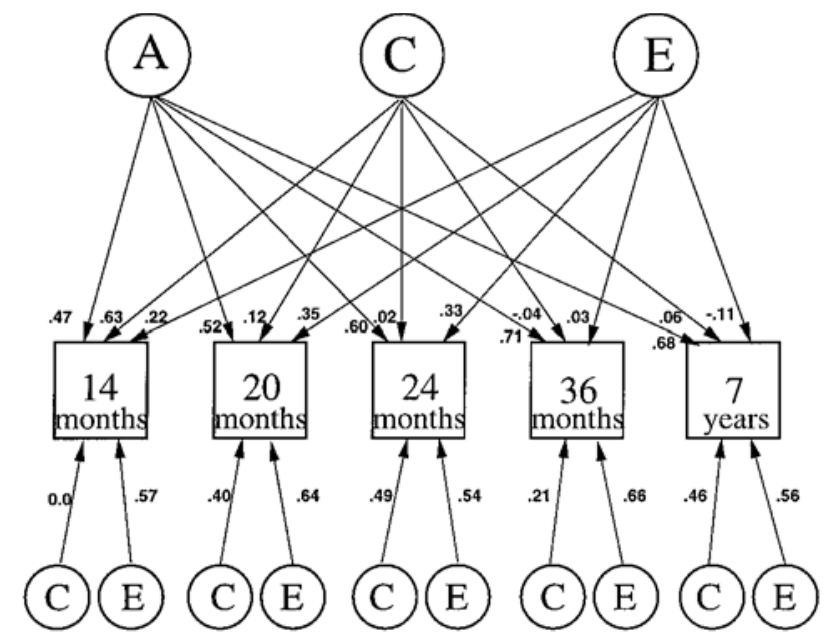

Figure 1 Independent pathways model of latent genetic, shared environmental, and non-shared environmental effects ( $A, C$ and $E$ respectively) common to all five ages as well as shared and nonshared environmental effects specific to each age

behavioral characteristics such as childhood inhibition and delinquency. ${ }^{4,27}$ Whether or not heart rate really is indicative of behavior remains in question. For instance, Raine et al $^{28}$ found that resting heart rate measured as early as age 36 months predicted deliquency at age 11 years; however, Van Hulle et $\mathrm{al}^{29}$ failed to replicate the predictive relationship between heart rate and later delinquent behavior up to age 7 years. A better understanding of the underlying genetic and environmental influences on heart rate over time would strengthen an analysis of the association between heart rate and behavior. This is one of the few studies to examine the etiology of heart rate over time and confirms that individual differences in resting heart rate are indeed stable from infancy to middle childhood and genetic factors are primarily responsible for this stability. Although shared environment al so contributes to the stability of individual differences, such factors con-

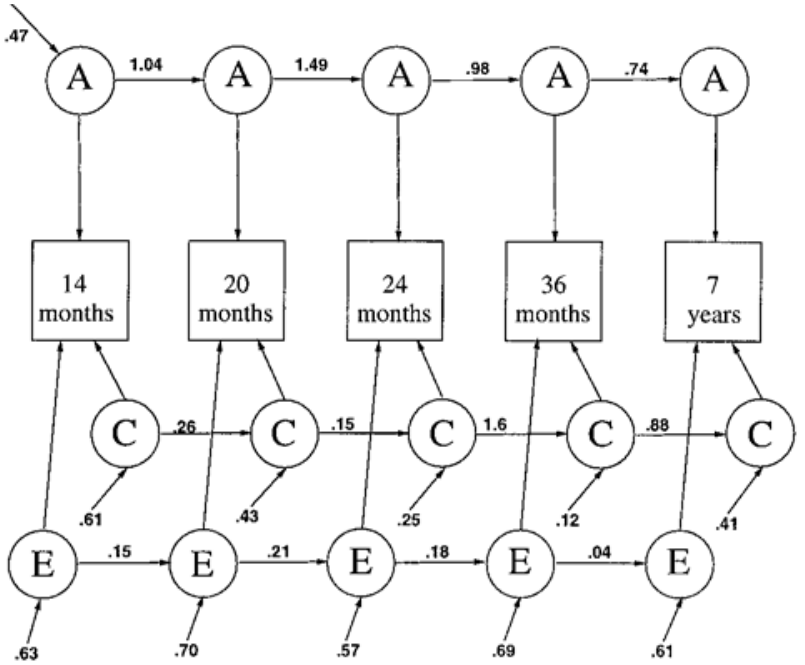

Figure2 Simplex model of genetic, shared environmental, and non-shared environmental effects (A, C, E respectively) on heart rate at 5 ages. The age specific effects are represented by unlabeled arrows

tribute more towards individual variation at specific ages. Non-shared environment contributes substantially to the individual variation at each age but contributes marginally to individual differences across ages.

\section{Acknowledgements}

This work was supported by a grant from the John D and Catherine $T$ MacArthur Foundation and partially supported by NIDA P60 Center grant DA-11015. The report was prepared while CA Van Hulle was supported by NICHD training grant HD-07289. The authors wish to thank Dr Tari Topolski and Corrine Wright for their help in processing the heart rate data.

Table 6 Estimated standardized variance components

\begin{tabular}{|c|c|c|c|c|c|}
\hline & 14 months & 20 months & 24 months & 36 months & 7 years \\
\hline \multicolumn{6}{|l|}{ Independent Pathways ${ }^{a}$} \\
\hline Genetic & $0.22(100 \%)$ & $0.27(100 \%)$ & $0.36(100 \%)$ & $0.50(100 \%)$ & $0.46(100 \%)$ \\
\hline Shared environment & $0.40(100 \%)$ & $0.17(8.5 \%)$ & $0.24(0.01 \%)$ & $0.05(3.2 \%)$ & $0.21(1.7 \%)$ \\
\hline Non-shared environment & $0.37(13.1 \%)$ & $0.53(23.1 \%)$ & $0.40(27.9 \%)$ & $0.44(0.02 \%)$ & $0.30(3.3 \%)$ \\
\hline \multicolumn{6}{|l|}{ Simplex ${ }^{b}$} \\
\hline Genetic & 0.22 & $0.24(100 \%)$ & $0.53(100 \%)$ & $0.51(100 \%)$ & $0.28(100 \%)$ \\
\hline Shared environment & 0.37 & $0.21(12.0 \%)$ & $0.09(5.2 \%)$ & $0.17(83.0 \%)$ & $0.32(46.2 \%)$ \\
\hline Non-shared environment & 0.40 & $0.50(1.7 \%)$ & $0.35(6.2 \%)$ & $0.49(2.3 \%)$ & $0.38(2.1 \%)$ \\
\hline
\end{tabular}

aNumbers in parentheses indicate the proportion of each component common across ages for the independent pathways model.

${ }^{b}$ For the simplex model numbers in parentheses indicate the proportion of each component contributed from the previous age. 


\section{References}

1 Scarpa A, Raine A, Venables PH, Sarnoff SA. Heart rate and skin conductance in behaviorally inhibited Mauritian children. J Abnorm Psychol 1997; 106(2): 182-190.

2 Farrington DP. The relationship between low resting heart rate and violence. In: Raine A, Brennan, PA, Farrington DP, Mednick SA (eds). Biosocial Bases of Violence. Plennum Press: New York, pp 89-105.

3 Garcia-Coll C, Kagan J, Reznick SJ. Behavioral inhibition in young children. Child Devel 1984; 55(3): 1005-1019.

4 Raine A. The Psychopathology of Crime: Criminal Behavior as a Clinical Disorder. Academic Press: San Diego, 1993.

5 Lacey J, Lacey B. The law of initial value in the longitudinal study of autonomic constitution: Reproductibility of autonomic responses and response patterns over a four-year interval. Ann N Y Acad Sci 1962; 98: 1257-1290.

6 Veit R, Brody S, Rau H. Four-year stability of cardiovascular reactivity to psychological stress. J Behav Med, 1997; 20(5): 447-460.

7 Mathers KA, Woodall KL, Stoney CM. Changes in and stability of cardiovascular responses to behavioral stress: Results from a four year longitudinal study of children. Child Devel, 1990; 61(4): 1134-1144

8 Fracasso M, Porges SW, Lamb ME, Rosenberg AA. Cardiac activity in infancy: Reliability and stability of individual differences. Infant Behav Devel 1994; 17: 277-284.

9 Wise NB, Comeau WJ, White PD. An electrocardiographic study of twins. Am Heart J 1939; 17: 701-710.

10 Perez GL, Keith L. El ectrocardiograms of twins. In: Nance WE, Allen G, Parisi $P$ (eds). Twin Research: Progress in Clinical Studies. Alan R Liss: New York, 1978; vol 24C: pp 39-47.

11 Ditto B. Sibling similarities in cardiovascular reactivity to stress. Psychophysiology 1987; 24(3): 353-360.

12 Sims J, Carroll D, Hewitt $K$, Turner R. Genetic and environmental determinants of heart rate variability: A twin study. Pyschophysiology 1990; 27: S63.

13 Russell MW, law I, Sholinsky P, Fabsitz RR. Heritability of ECG measurements in adult male twins. JElectrocardiol 1998; 30: 64-68.

14 Singh J, Larson MG, O'Donnel C, Tsuji H, Evans JC, Levy D. Heritability of heart rate variability: the Framingham Heart Study. Circulation 1999; 99(17): 2251-2254.
15 Havlik RJ, Garrison RJ, Fabsitz MA, Feinleib M. Variability of heart rate, P-R, QRS and Q-T duration in twins. J Electrocardiol 1980; 3: 45-48.

16 Hanson B, Tuna N, Bouchard T, Heston L, Eckert E, Lyken D, Sega N, Rich S. Genetic factors in the el ectrocardiogram and heart rate of twins reared apart and together. Am J Cardiol 1989; 63: 606-609.

17 Ditto B. Familial influences on heart rate, blood pressure, and self-report anxiety responses to stress: Results from 100 twin pairs. Psychophysiology 1993; 30: 635-645.

18 Schieken RM, Eaves LJ, Hewitt JK, Mostellar M, Bodurtha N Moskowitz WB, Nance WE. Univariate genetic analysis of blood pressure in children: The Medical College of Virginia Twin Study. Am J Cardiol 1989; 64: 1333-1337.

19 Boomsma DI, Gabrielli WF. Behavioral genetic approaches to psychophysiology data. Psychophysiology 1985; 22(3): 249-260.

20 Boomsma DI, van den Bree M, Orlebeke F. Resemblances of parents and twins in sports participation and heart rate. Behav Genet 1989; 19(1): 123-141.

21 Anderson NB, McNeilly M. Age, gender, and ethnicity as variables in psychophysiological assessment: Sociodemographics in context. Psychol Assess 1992; 3(3): 376-384.

22 Milnor WR. Aortic wavel ength as a determinant of the relation between heart rate and body size in mammals. Am J Physiol 1979; 237(1): R3-6.

23 Cardon LR. Height, weight, and obesity. In: De Fries JC, Plomin R, Fulker DW (eds). Nature and Nurture during Middle Childhood. Blackwell: Cambridge, MA, 1994, pp 165-172.

24 Nichols RC, Bilbro WC. The diagnosis of twin zygosity. Acta Genet Med Stat 1966; 16: 265-275.

25 Cole PM, Jordan PR, Zahn-Waxler C. Mood Induction Stimulus for Children. National Institute of Mental Health: Bethesda, MD, 1990

26 Neale MC. Mx: Statistical Modeling 5th edn. MCV Department of Psychiatry: Richmond, VA, 1999.

27 Rosenberg AA, Kagan J. Physical and physiological correlates of behavorial inhibition. Devel Psychol 1989; 22(8): 753-770.

28 Raine A, Venables PH, Mednick SA. Low resting heart rate at age 3 years predisposes to aggression at age 11 years: Evidence from the Mauritius Child Health Project. J Am Acad Child Adolesc Psychiatry 1997; 36(10): 1457-1464.

29 Van Hulle CA, Corley R, Zahn-Waxler C, Kagan J, Hewitt J. Early childhood heart rate does not predict externalizing behavior at age seven years. J Am Acad Child Adolesc Psychiatry in press. 